\title{
Endocannabinoid-Mediated Long-Term Depression in the Avian Midbrain Expressed Presynaptically and Postsynaptically
}

\author{
Mario Alexander Penzo and José Luis Peña \\ Dominick P. Purpura Department of Neuroscience, Albert Einstein College of Medicine, Bronx, New York 10461
}

\begin{abstract}
Here, we examined long-term synaptic plasticity in the avian auditory midbrain, a region involved in experience-dependent learning. We found that coactivation of $N$-methyl-D-aspartate receptors (NMDAR) and type 1 cannabinoid receptors (CB1R) induces long-term depression (LTD) at the synapse between the central shell and the external portion of the inferior colliculus of the chicken. Although endocannabinoids are commonly thought of as presynaptic modulators, recent reports have suggested that they can also modulate the postsynaptic site. In the avian midbrain, we found that LTD is mediated by both presynaptic and postsynaptic changes. The presynaptic mechanism consists of a decrease in neurotransmitter release, whereas a depression of NMDAR-mediated current takes place on the postsynaptic side. Both the presynaptic and the postsynaptic effects depend on CB1R activation. The reduction of postsynaptic NMDAR currents represents a novel role of endocannabinoids in synaptic modulation.
\end{abstract}

\section{Introduction}

The auditory midbrain is attractive to study synaptic plasticity because it has been involved in experience-dependent learning of auditory spatial cues in birds and mammals (King et al., 1988; Knudsen and Knudsen, 1989). However, there have been few studies on long-term synaptic plasticity in this region of the brain (Hosomi et al., 1995; Zhang and Wu, 2000; Wu et al., 2002). We studied neurons in the external part of the midbrain auditory torus (EX) of the chicken, which is the external section of the nucleus mesencephalicus lateralis pars dorsalis (MLd) (Puelles et al., 2007). Early work has shown that neurons in the chicken MLd respond to auditory spatial cues (Coles and Aitkin, 1979). In barn owls, EX is the site where visually instructed modulation of the auditory spatial tuning takes place (Brainard and Knudsen 1993). EX is therefore a good candidate for the study of synaptic plasticity. We used late chicken embryos to examine activity-dependent modulation at the synaptic connection between EX cells and their afferent input, which originate in the central shell part of the midbrain auditory torus (CSh) (Puelles et al., 2007). In this synapse, we observed a form of LTD mediated by endocannabinoids (eCB).

Induction of most eCB-mediated LTDs depends solely on the activation of type 1 cannabinoid receptors (CB1R), whereas others require coactivation of both presynaptic $N$-methyl-D-

Received Nov. 12, 2008; revised Feb. 19, 2009; accepted Feb. 25, 2009.

This work was supported by National Institutes of Health Grants DC007690 and DC007690.S1. We thank Pablo Castillo, Reed Carroll, Alberto Pereda, and Diana Pettit for their comments and advice, and María Lucía Pérez and Andrea Reyna for their help with immunohistochemistry.

Correspondence should be addressed to José L. Peña, Dominick P. Purpura Department of Neuroscience, Albert Einstein College of Medicine of Yeshiva University, 1410 Pelham Parkway, Bronx, NY 10461. E-mail: jpena@aecom.yu.edu.

DOI:10.1523/JNEUROSCI.5466-08.2009

Copyright $\odot 2009$ Society for Neuroscience $\quad$ 0270-6474/09/294131-09\$15.00/0 aspartate receptors (NMDAR) and CB1R (Sjöström et al., 2003; Bender et al., 2006). These forms of LTD are expressed presynaptically (Chevaleyre et al., 2006). However, one group has shown that CB1R activation is necessary for the induction of a postsynaptically expressed LTD (Safo and Regehr, 2005). Despite the apparent ability of eCBs to modulate both sites of the synapse, it is unclear whether coincident presynaptic and postsynaptic eCBmediated LTD occurs in the brain.

We report here the first evidence of long-term synaptic plasticity observed at the external portion of the avian inferior colliculus. Unlike previously described eCB-LTD, this phenomenon is expressed both at the presynaptic and postsynaptic sites. We identified a novel effect of eCBs, consisting of a reduction in postsynaptic NMDAR current. This effect may alter the ability of NMDAR signaling to modulate synaptic function.

\section{Materials and Methods}

Slice preparation. We used chicken embryos from embryonic day 17 (E17) to E19. Slices were prepared by making brain sections parallel to the dorsoventral axis of the optic lobe. Slices were then incubated for $1 \mathrm{~h}$ at $38^{\circ} \mathrm{C}$ in artificial CSF (ACSF) containing (in mM): $139 \mathrm{NaCl}, 2.9 \mathrm{KCl}$, $1 \mathrm{MgSO}_{4}, 3.14 \mathrm{CaCl}_{2}, 17 \mathrm{NaHCO}_{3}$, and $12.2 \mathrm{D}$-glucose, bubbled with $95 \% \mathrm{O}_{2}$ and $5 \% \mathrm{CO}_{2}$, and $\mathrm{pH}=7.4$. The same solution was used as recording solution.

Electrophysiology. Whole-cell patch-clamp recordings of EX neurons were performed at $38^{\circ} \mathrm{C}$ (Temperature Controller, Warner Instrument) using a MultiClamp 700B amplifier (Axon Instruments). The recording electrodes were filled with a solution containing (in mM): $110 \mathrm{~K}$ gluconate, $10 \mathrm{HEPES}, 10 \mathrm{NaCl}, 10$ phosphocreatine (dipotassium salt), 0.2 EGTA, 5 ATP (dimagnesium salt), 0.4 GTP (trisodium salt) and 10 glucose, $\mathrm{pH}$ 7.2. In all experiments picrotoxin $(100 \mu \mathrm{M})$ was added to the ACSF used for perfusion during recording, to block $\mathrm{GABA}_{\mathrm{A}}$ receptors. In a set of experiments (in $\mu \mathrm{M}$ ) $50 \mathrm{D}$-AP5 or $100 \mathrm{D}-\mathrm{CPP}$ were used to block NMDAR, and 25 DL-TBOA, 2 A.M.-251 or 5 SR-141716A and 120 LY367385 were used to block glutamate uptake, CB1R and mGluR1/5, 
respectively. WIN55212-2 (5 $\mu \mathrm{M})$ and DHPG $(50 \mu \mathrm{M})$ were used as CB1R and mGluR1/5 agonists. Intracellular dialysis with MK-801 (1 mM) was used to block postsynaptic NMDARs. BAPTA (10 mM) was applied to sequester intracellular postsynaptic calcium. $\mathrm{G}_{\mathrm{i} / \mathrm{o}}$ blocker pertussis toxin (PTX, $200 \mathrm{ng} / \mathrm{ml}$ ) and the PKA inhibitor PKI 6-22 (10 $\mu \mathrm{M})$ were used to block $\mathrm{G}_{\mathrm{i} / \mathrm{o}}$ and PKA, respectively. PTX was prepared from a stock solution containing $50 \mu \mathrm{g}$ of the protein in 500 $\mu \mathrm{l}$ of a $0.01 \mathrm{M}$ sodium phosphate buffer and $0.05 \mathrm{M}$ sodium chloride solution at $\mathrm{pH} 7.0$, as indicated by the provider (Tocris). Other than the standard ATP used for the recording-electrode solution, no supplemental activators were used during intracellular dialysis with PTX. This is consistent with the manufacturer's statement that activation is only required in cellfree media and to a careful analysis of the literature which indicates that the pertussis toxin is activated on entrance to the cell by an ATP-dependent mechanism (for review see Kaslow and Burns 1992). GDP- $\beta S$ (2 $\mathrm{mM}$ ) was used as a broad spectrum G-protein blocker. ACSF chemicals were obtained from Sigma-Aldrich. All drugs were obtained from Tocris except for GDP$\beta S$, which was bought from BIOMOL.

EX neurons were recorded in voltage clamp configuration. Currents were low-pass filtered at $3 \mathrm{kHz}$ and acquired with $5 \mathrm{kHz}$ sampling rate using Igor Pro software (WaveMetrics). Membrane potentials were held within -65 to $-75 \mathrm{mV}$. Input resistance and series resistance were monitored throughout each experiment with test pulses of $10 \mathrm{mV}$ and $80 \mathrm{~ms}$ duration at the beginning of each trace.

For pathway stimulation, patch-type pipettes of larger tip size $(10-20 \mu \mathrm{M})$ were filled with saline. The position of the stimulating electrode in CSh was determined by first localizing the central core part of the midbrain auditory torus (CCo), which is easily identifiable visually as a denser area; CSh is located in between CCo and EX (Fig. $1 A$ ). Electrical stimulation consisted of pulses of $50-300 \mu \mathrm{A}$ and $0.1 \mathrm{~ms}$ duration delivered to CSh every $5 \mathrm{~s}$.

Experiments in which the series resistance changed by $>20 \%$ were excluded from our study. We measured the latency and rise time of the EPSCs evoked CSh stimulation. The latency was determined by measuring the time between the peak of the stimulation artifact and the response peak. The rise time was assessed as the time interval between the $20 \%$ and the $80 \%$ of the EPSCs' peak amplitude. Only synaptic responses that exhibited latency and rise time values consistent with monosynaptic connections were used (see Results).

LTD at CSh-EX synapses was induced after obtaining a stable baseline with CSh stimulation at $0.2 \mathrm{~Hz}$ for 5 min or more. The high-frequency stimulation (HFS) protocol consisted of four trains of one hundred pulses at $100 \mathrm{~Hz}$ with an intertrain interval of 5-7 s under current clamp configuration. The lowfrequency stimulation (LFS) protocol consisted of paired stimulation of CSh at $1 \mathrm{~Hz}$ (interstimulus interval of $40 \mathrm{~ms}$ ) for 4 min. The HFS protocol was chosen after pilot experiments showed that a classical LFS protocol did not induce LTD in this synapse (see Results).

NMDA/AMPA ratios were measured as follows: neurons were hyperpolarized to $-80 \mathrm{mV}$ and CSh was stimulated at $0.2 \mathrm{~Hz}$ to determine the timing of the AMPA current. Then, NMDA/AMPA ratios were calculated by measuring the amplitude of both the AMPAR and NMDAR components at $+50 \mathrm{mV}$ to reduce $\mathrm{Mg}^{2+}$ blockade of the NMDAR. The time selected to measure the AMPA component was determined at -80 $\mathrm{mV}$, whereas the NMDAR component was measured $20 \mathrm{~ms}$ after the
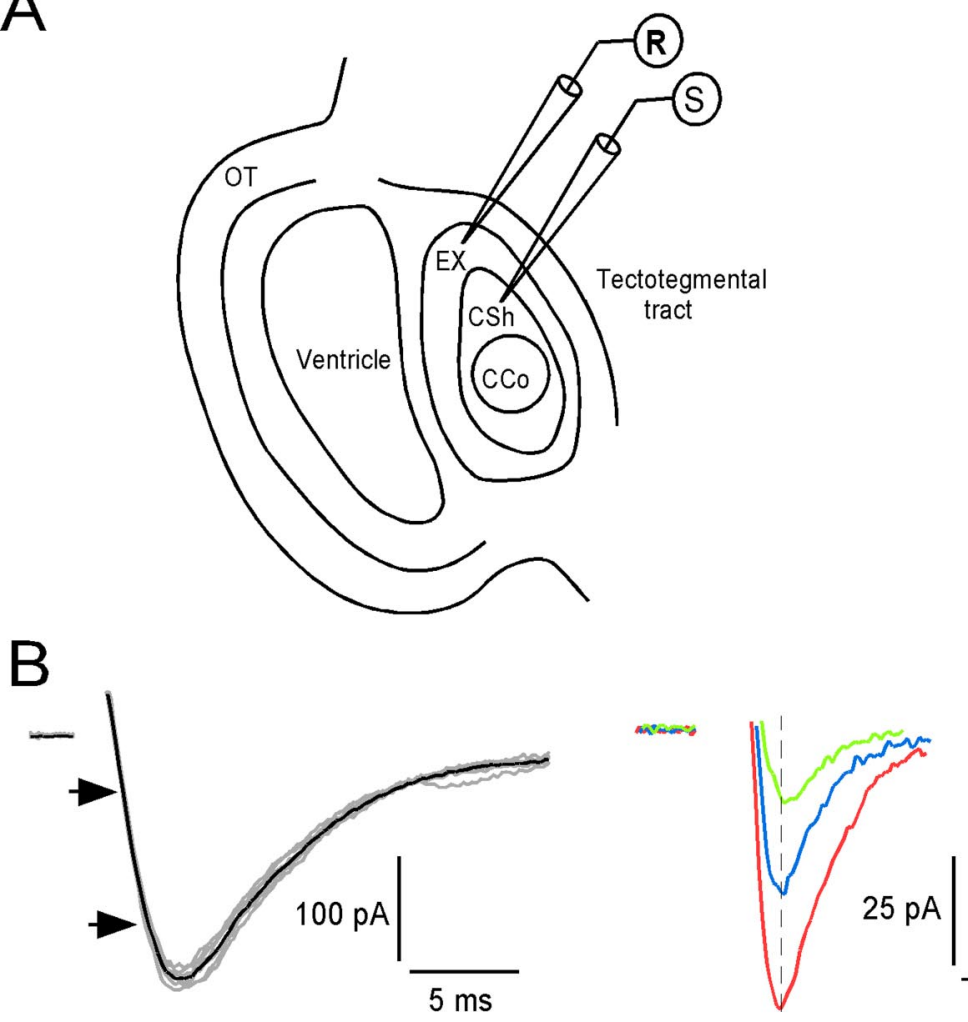

$10 \mathrm{~ms}$

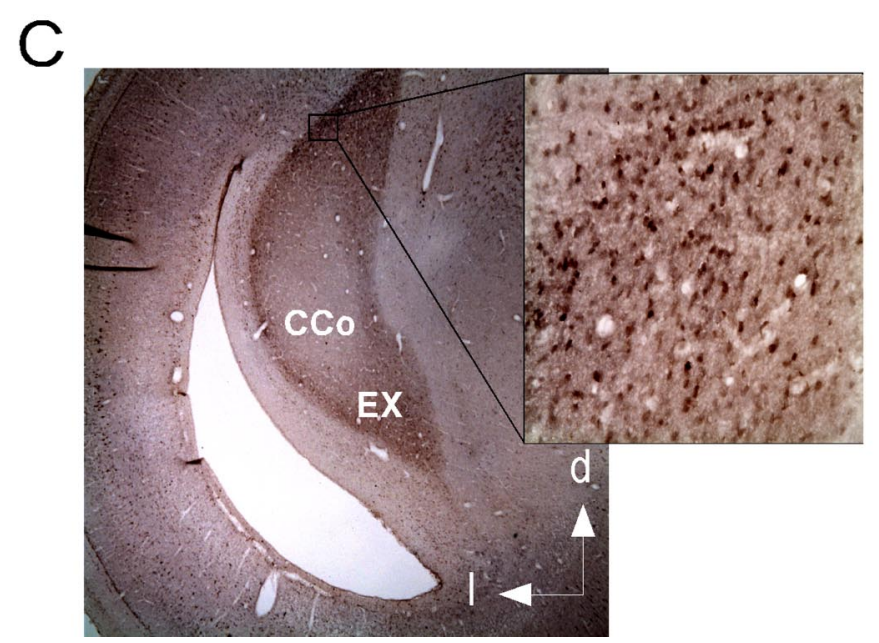

Figure 1. Identification of the EX. $A$, Schematic of a coronal section of the chicken midbrain showing the recording $(R)$ and the stimulation (S) sites. $\boldsymbol{B}$, Left, Ten overlaid EPSCs evoked by stimulation of the CSh (gray traces) and their average (black trace). Arrows indicate the interval over which the rise time of the synaptic current was measured. $\boldsymbol{B}$, Right, Three overlaid EPSCs (each trace is an average of six trials) evoked by $10 \mathrm{~Hz}$ stimulation of (Sh. Red, blue, and green represent stimuli 1, 2, and 3 respectively. The dashed line passes through the peak of the largest EPSC, for time reference. C, Immunohistochemistry for $\alpha$ CaMKII selectively labels EX neurons' cell bodies (inset), which lie around the $(C 0$. Slice shown at $4 \times$ magnification (inset at $40 \times$ ). d, Dorsal; I, lateral; $0 \mathrm{~T}$, optic tectum.

stimulus onset. At this point AMPA responses decayed almost completely (9095\%) and the amplitude of the NMDA responses could be estimated with minimal noise. In addition, synaptic responses from experiments performed with bath application of AP5 showed full decay by $20 \mathrm{~ms}$. In a subset of experiments NMDAR and AMPAR components were both measured at $-10 \mathrm{mV}$ (indicated in the text).

Immunohistochemistry. Chicken embryos age E21 were perfused transcardially with $100 \mathrm{ml}$ of $0.1 \mathrm{M}$ phosphate buffer (PB), followed by $200 \mathrm{ml}$ of $4 \%$ paraformaldehyde in PB (fixative), and then $100 \mathrm{ml}$ of $10 \%$ sucrose in fixative (all ice-cold). The brain was removed, immersed in $30 \%$ sucrose in $\mathrm{PB}$ at $4^{\circ} \mathrm{C}$ 

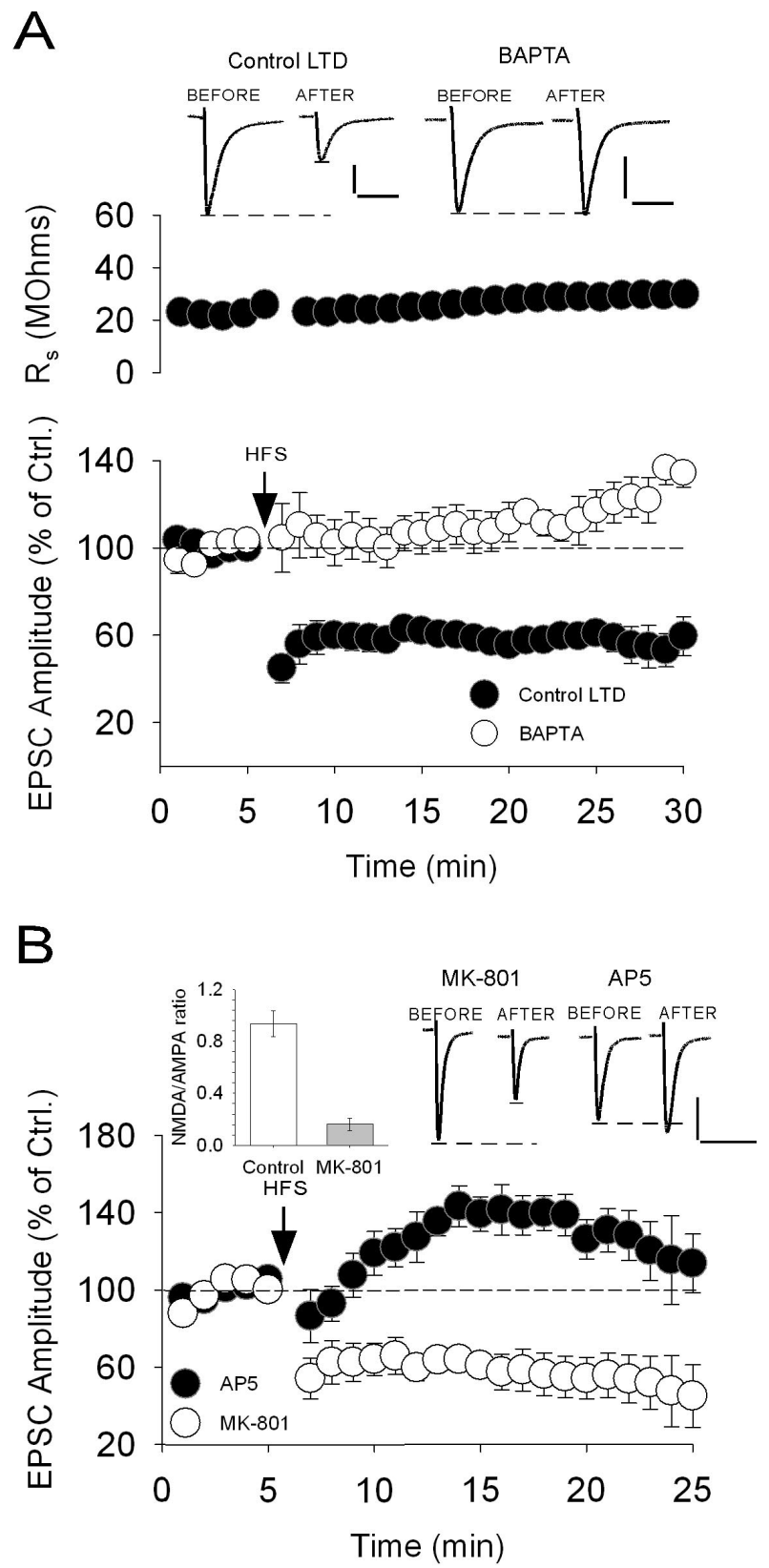

$\mathrm{C}$

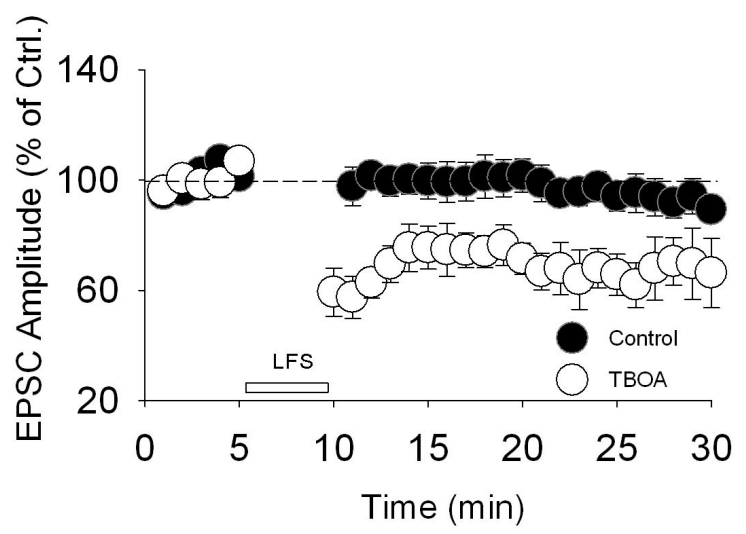

Figure 2. LTD induction by HFS in CSh. $A$, Four trains of 100 pulses at $100 \mathrm{~Hz}$ (HFS, arrow) applied to CSh induces LTD (black circles). Postsynaptic dialysis with calcium chelator BAPTA (10 $\mathrm{mm}$ ) blocks LTD induction (white circles). The traces above the plot show example traces $2 \mathrm{~min}$ overnight, for cryoprotection. Midbrain sections $(25 \mu \mathrm{m})$ parallel to the dorsoventral axis of the optic tectum were cut with a cryostat. Sections were incubated in a blocking solution containing (in \%): 4.0 normal goat serum (Vector Laboratories, Burlingame, CA), 0.4 Triton X-100, and 1.0 bovine serum albumin (BSA; Sigma, St. Louis, MO) for $1 \mathrm{~h}$ at room temperature. Primary antibodies were diluted in 1.0 normal goat serum, 0.3 Triton X-100, and 1.0 BSA. The antibody used was anti- $\alpha$ CaMKII at 1:100 dilution (clone 6G9, subtype IgG1; Millipore). The tissue was incubated in primary antibody for $24-48 \mathrm{~h}$ at $4^{\circ} \mathrm{C}$ under gentle agitation. After primary antibody incubation, the tissue was rinsed two times for 5 min in 0.02 Triton X-100 and 0.25 BSA at room temperature. Sections were later incubated at $4^{\circ} \mathrm{C}$ for $12-24 \mathrm{~h}$ in a solution containing biotinylated anti-mouse diluted at 1:200 in 0.02 Triton X-100 and 1.0 BSA as secondary antibody. Before mounting, the tissue was rinsed five times for $2 \mathrm{~min}$ in $0.25 \mathrm{BSA}$ and two times for $5 \mathrm{~min}$ in PB. Tissue sections were mounted, dehydrated, and then coverslipped using Permount (Fisher Scientific). All dilutions were made in $0.1 \mathrm{M} \mathrm{PB}$, except where indicated.

\section{Results}

We performed whole-cell patch clamp of visually identified EX neurons in acute midbrain slices (Fig. 1A). Antibodies directed against $\mathrm{Ca}^{2+} /$ calmodulin-dependent protein kinase II $\alpha$ (CaMKII) exclusively label neurons of EX in barn owls (Rodriguez-Contreras et al., 2005). In pilot experiments, we identified the EX as the lateral portion of MLd (the chicken equivalent to the inferior colliculus), which also contains neurons positive to CaMKII (Fig. 1C). EPSCs were evoked by lowintensity electrical stimulation of CSh (Fig. $1 \mathrm{~B}$ ), which lies medial to EX (Puelles et al., 2007). All experiments were performed in the presence of $100 \mu \mathrm{M}$ of the GABA blocker picrotoxin. Synaptic responses had short peak latency $(3.5 \pm 0.4 \mathrm{~ms})$ and $20-80 \%$ amplitude rise time of $0.8 \pm 0.2 \mathrm{~ms}$. Their amplitude decreased uniformly during trains of stimuli at moderate frequency (10 $\mathrm{Hz}$ ), without change in peak latency, which is consistent with activation of monosynaptic connections (Fig. $1 B$ ).

HFS of the CSh afferents to EX consistently produced LTD (Fig. $2 A$, black circles) $(n=8)$. LTD was blocked by intracellular dialysis with a low concentration $(10 \mathrm{mM})$ of the calcium chelator BAPTA (Fig. $2 A$, white circles) $(n=5)$, indicating that this form of synaptic plasticity requires postsynaptic $\mathrm{Ca}^{2+}$.

To elucidate the mechanism responsible for triggering LTD, we first examined the role of NMDARs. Bath application of D-AP5 $(50 \mu \mathrm{M})$ blocked LTD (Fig. $2 B$, black circles) $(n=5)$, suggesting that NMDARs are involved. To determine the locus of these NMDARs, we included the activity-dependent NMDARblocker MK-801 (1 mM) in the recording-pipette solution in a separate experiment. MK-801 eliminated postsynaptic NMDAR currents (Fig. $2 B$, inset) but did not block LTD (Fig. $2 B$, white circles) $(n=6)$. These results are consistent with nonpostsynaptic NMDARs mediating LTD.

Recent studies have linked LTD induced by HFS with glutamate spillout (Massey et al., 2004; Yang et al., 2005). Unlike HFS, low-frequency stimulation (LFS; one per second paired-pulse stimulation with a $40 \mathrm{~ms}$ interstimulus interval) of the CSh affer-

\footnotetext{
$\leftarrow$

before and 5 min after HFS with (right) and without (left) BAPTA in the pipette $(n=5)$. A representative recording of series resistance is shown on the top. $B$, Bath-applied D-AP5 (black circles) but not postsynaptic dialysis with the NMDA-blocker MK-801 (white circles) blocks LTD induction $(n=6)$. The bar histogram on the top left shows the NMDA/AMPA ratios of control (white bars) and with MK-801 application (gray bars), indicating that the NMDA component of the EPSC decreases by the effect of MK-801. The traces show example EPSCs, 2 min before and 5 min after HFS. C, Although LFS (white bar) alone does not induce LTD (black circles), LFS with simultaneous bath application of the glutamate uptake inhibitor TBOA does (white circles; $n=$ 5). Calibration: $100 \mathrm{pA}$ and $50 \mathrm{~ms}$.
} 
ents to EX did not induce LTD (Fig. 2C, black circles) $(n=6)$. To check whether a higher concentration of glutamate at the synaptic cleft was required for LTD, we tested the effect of LFS in the presence of the glutamate uptake inhibitor DL-TBOA $(25 \mu \mathrm{M})$. Under these conditions, LFS induced LTD (Fig. $2 C$, white circles) $(n=5)$. This is consistent with spillout glutamate reaching nonpostsynaptic, presumably presynaptic, NMDARs.

To find out whether or not this LTD was expressed presynaptically, we examined pair-pulse ratio (PPR) before and after LTD induction. PPR has been extensively used as an indirect estimation of changes in release probability $(\rho)$ at synaptic terminals (Zucker and Regehr, 2002). A long-term increase in PPR was consistently observed after LTD induction, suggesting $\rho$ had decreased in the CSh terminals (Fig. $3 A)(n=6)$. To corroborate the decrease in $\rho$, we measured failure rates before and after LTD induction. In these experiments, we delivered weak focal stimulation $(10-30 \mu \mathrm{A})$ to CSh to activate only a small number of fibers. This stimulation produced failure rates from 10 to $30 \%$. HFS using the same weak stimulus significantly increased failure rates $(237 \pm 70 \%$ relative to the baseline, $p<0.003 ; n=6$ ) (Fig. 3B). Although a reduction in the mean EPSC amplitude (including failures) was observed after HFS ( $58 \pm 20 \%$ relative to the baseline, $p<0.02$ ), the potency of the synapse (mean EPSC amplitude without failures) was largely maintained (90 \pm $40 \%$ relative to the baseline). Together, these results indicate that LTD at CSh-EX synapse is caused by a long-lasting decrease in transmitter release.

As noted above, postsynaptic calcium is required to induce the presynaptic expression of this LTD. The observation that a presynaptic LTD requires postsynaptic signaling, suggests that a retrograde signal, synthesized in the postsynaptic compartment, instructs changes in the presynaptic site. Because postsynaptic calcium is required, we reasoned that eCBs could constitute such a signal. eCBs are synthesized postsynaptically from lipid precursors in both calciumdependent and calcium-independent processes (Wilson and Nicoll, 2002) and have been involved in presynaptic LTD (Chevaleyre et al., 2006). We tested the involvement of eCB by applying the LTD-induction protocol in the presence of AM-251 (2 $\mu \mathrm{M})$, a CB1R antagonist. AM-251 consistently blocked LTD induction (Fig. $4 A)(n=5)$, suggesting a role of CB1R in this process. To further explore whether CB1Rs were present at this synapse, we applied the CB1R agonist WIN55212-2 (WIN) to the bath solution $(5 \mu \mathrm{M})$. WIN decreased the amplitude of evoked synaptic currents (Fig. $4 B)(n=6)$. In a subset of experiments, subsequent application of the CB1R antagonist SR-141716A (SR; $5 \mu \mathrm{M}$ ) blocked the depression induced by WIN (Fig. $4 B)(n=3)$, indicating that the effect of WIN is caused by a transient activation of
CB1R and not a long-term one. Together, our results suggest that activation of both CB1Rs and presynaptic NMDARs is required to induce LTD in CSh-EX synapses. These results are the first evidence of the presence of CB1-like receptors in the avian inferior colliculus.

To identify the postsynaptic mechanism responsible for $\mathrm{eCB}$ release and subsequent LTD induction, we tested the involvement of metabotropic glutamate receptors (mGluRs). mGluR activation has been shown to induce eCB-mediated LTD in several preparations (see review by Chevaleyre et al., 2006). Bath application of the group I mGluR antagonist LY $367385(120 \mu \mathrm{M})$ blocked LTD induction (Fig. 4C) $(n=7)$. In addition, application of the mGluR1/5 agonist DHPG $(50 \mu \mathrm{M})$ induced depression (Fig. $4 D$, black circles) $(n=4)$, expressed as a decrease in $\rho$ (data not shown). This depression was blocked by bath application of either AM-251 (Fig. $4 D$, white circles) $(n=4)$ or D-AP5 (Fig. $4 D$, gray circles) $(n=5)$. However, once established, the effect of DHPG was not affected by either LY 367385 (Fig. 4E) $(n=3)$ or 

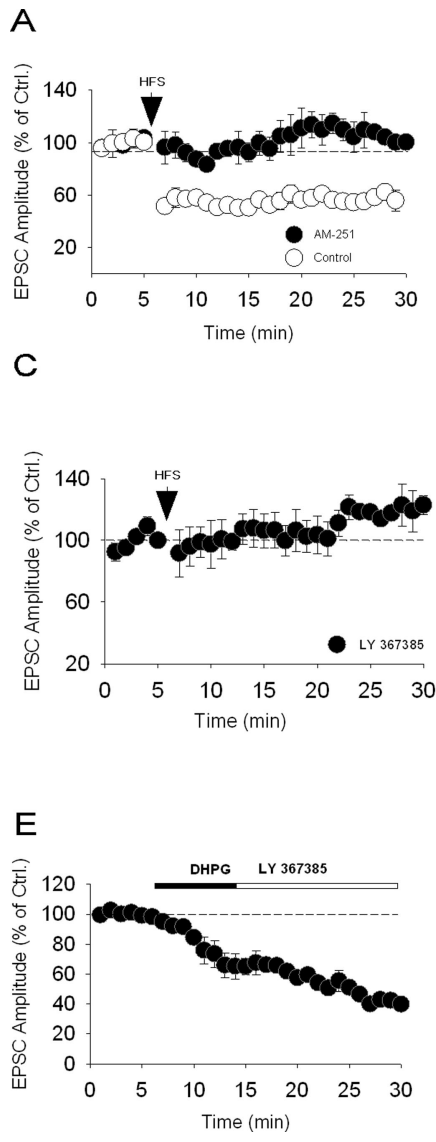

Figure 4. Involvement of CB1Rs and mGluRs in LTD. A, The CB1R antagonist AM-251 applied to the bath blocks LTD induction by HFS (black circles; $n=5$ ). White circles represent control experiments performed concomitantly $(n=3)$. $\boldsymbol{B}$, Bath application of the CB1R agonist WIN reduces synaptic responses by almost 50\% (black circles). Synaptic responses recover if WIN application is followed by the (B1R antagonist SR-141716A (white circles). PPR values before and after WIN and SR application are shown on the right. Horizontal bars indicate the time course of WIN (black) and SR-141716A (white) application. C, The mGluR1/5 antagonist LY367385 blocked LTD induction $(n=3)$. D, The mGluR1/5 agonist DHPG applied to the bath induces depression (black circles, $n=3$ ), which can be blocked by the (B1R antagonist AM-251 previously applied to the bath (white circles, $n=3$ ). A representative plot of series resistance is shown on top. PPR values before and after DHPG application are shown on the right. $\boldsymbol{E}, \boldsymbol{F}, 0$ nce the depression by DHPG is expressed, neither LY367385 alone $(\boldsymbol{E} ; n=3)$ nor LY367385 and AM-251 applied together $(\boldsymbol{F} ; n=3)$ can block this effect.

by LY 367385 and AM-251 applied together (Fig. $4 F)(n=3)$. These results indicate that, mGluR1/5 are both necessary and sufficient to produce LTD, mediated by eCB and NMDAR activation. Moreover, these results show that a transient exposure to eCB is sufficient to express mGluR-induced LTD.

Our data indicate that LTD is presynaptically expressed at this synapse. However, experience-dependent plasticity in barn owls has been associated with changes in the ratio between responses mediated by NMDA and non-NMDA receptors (Feldman and Knudsen, 1998). We therefore tested whether postsynaptic NMDAR currents were selectively modified over AMPAR currents during LTD. Such change would not be evident holding the cells at $-65 \mathrm{mV}$, a potential at which magnesium blocks most NMDAR current. Thus, both NMDAR and AMPAR components were monitored simultaneously by holding the cell's membrane potential at $-10 \mathrm{mV}$ throughout the entire recording. We found that both NMDAR and AMPAR currents decreased after LTD induction, as expected by the reduction in glutamate release. However NMDAR currents were significantly more depressed than AMPA currents (Fig. $5 A)(n=5$, paired $t$ test, $p<0.0001)$. The NMDA to AMPA ratios were similar before $(0.68 \pm 0.09 ; n=$ $4)$ and after $(0.31 \pm 0.07)$ LTD induction, and before $(0.72 \pm$
$0.13 ; n=4)$ and after $(0.26 \pm 0.05)$ bath application of D-AP5. Furthermore, mGluR1/5 activation by DHPG elicited a sustained depression of the NMDAR component of mixed NMDA/AMPA currents monitored at $-10 \mathrm{mV}$ (Fig. $5 B)(n=5)$. These observations suggest that, in addition to the decrease in release probability, there is a decrease in postsynaptic NMDAR currents.

NMDARs are known to have a higher affinity to glutamate than AMPARs (Patneau and Mayer, 1990). This property could render NMDARs more responsive to glutamate spillout. Thus, it is possible that the reduction in NMDAR currents is a side-effect of the decrease in glutamate concentration at the synaptic cleft, induced by the reduction in neurotransmitter release. To investigate this possibility, we tested postsynaptic NMDAR response to glutamate by focal application of $5 \mathrm{~mm}$ glutamate onto EX neurons with a PicoSpritzer, in the presence of CNQX to block AMPA currents. After obtaining a stable baseline for $18 \mathrm{~min}$, DHPG was applied. This allowed us to induce LTD without activation of the presynaptic compartment. We found that DHPG reduced postsynaptic NMDAR currents by almost 30\% (Fig. $5 C$, white symbols) $(n=4)$. In a subset of experiments the NMDAR antagonist 3-(2-carboxypiperazin-4-yl) propyl-1phosphonic acid (CPP) was added at the end, to confirm that there was no residual component left unblocked. These results further show that a reduction in postsynaptic NMDAR currents contributes to the LTD.

We then searched for the mediators of the postsynaptic decrease of NMDAR activity triggered by LTD induction. Because postsynaptic effects of eCB-mediated LTD have been reported (Safo and Regehr, 2005), we tested whether the reduction in the NMDAR component was also eCB-dependent. NMDA to AMPA ratios before $(0.48 \pm$ $0.04 ; n=3)$ and after $(0.51 \pm 0.07)$ HFS in the presence of AM-251 were not significantly different. In addition, the effect of DHPG on postsynaptic NMDAR currents was prevented by bath application of AM-251 (Fig. $5 C$, black symbols) $(n=6)$. In fact, the amplitude of NMDAR currents increased. These results suggest that CB1Rs play a role in modulating postsynaptic NMDARs in the EX.

Because the effect of DHPG on postsynaptic NMDAR currents induced by focal application of glutamate is CB1Rdependent, we reasoned that postsynaptic CB1Rs could mediate it. We then tested whether pharmacological activation of CB1Rs could mimic the reduction in postsynaptic NMDAR currents induced by DHPG. Bath application of the CB1R agonist WIN55212-2 decreased postsynaptic NMDAR currents, measured with focal application of glutamate onto EX neurons and AMPA receptors blockade (Fig. $6 A)(n=5)$. To examine whether the CB1Rs involved in decreasing NMDAR currents could be present on the postsynaptic side, we performed intracellular di- 


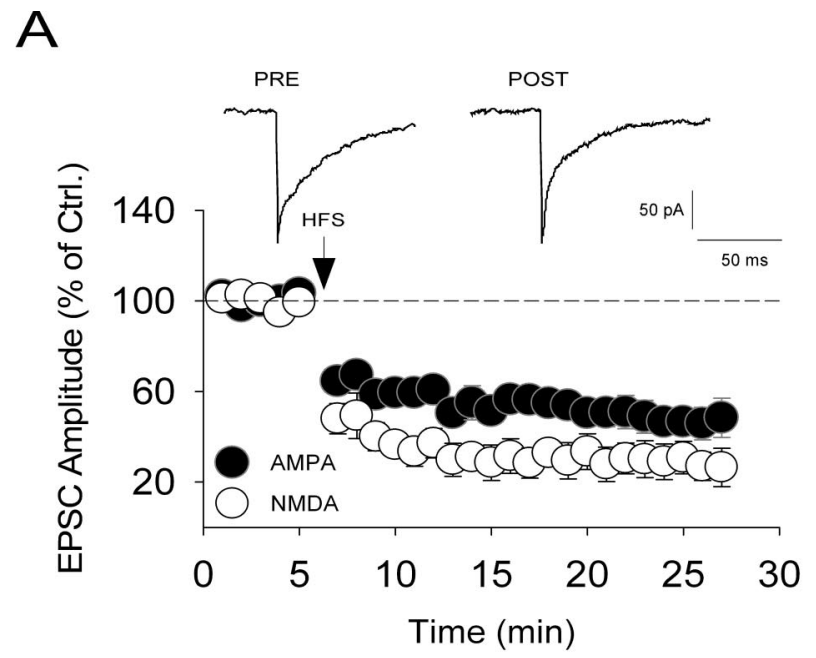

$\mathrm{B}$
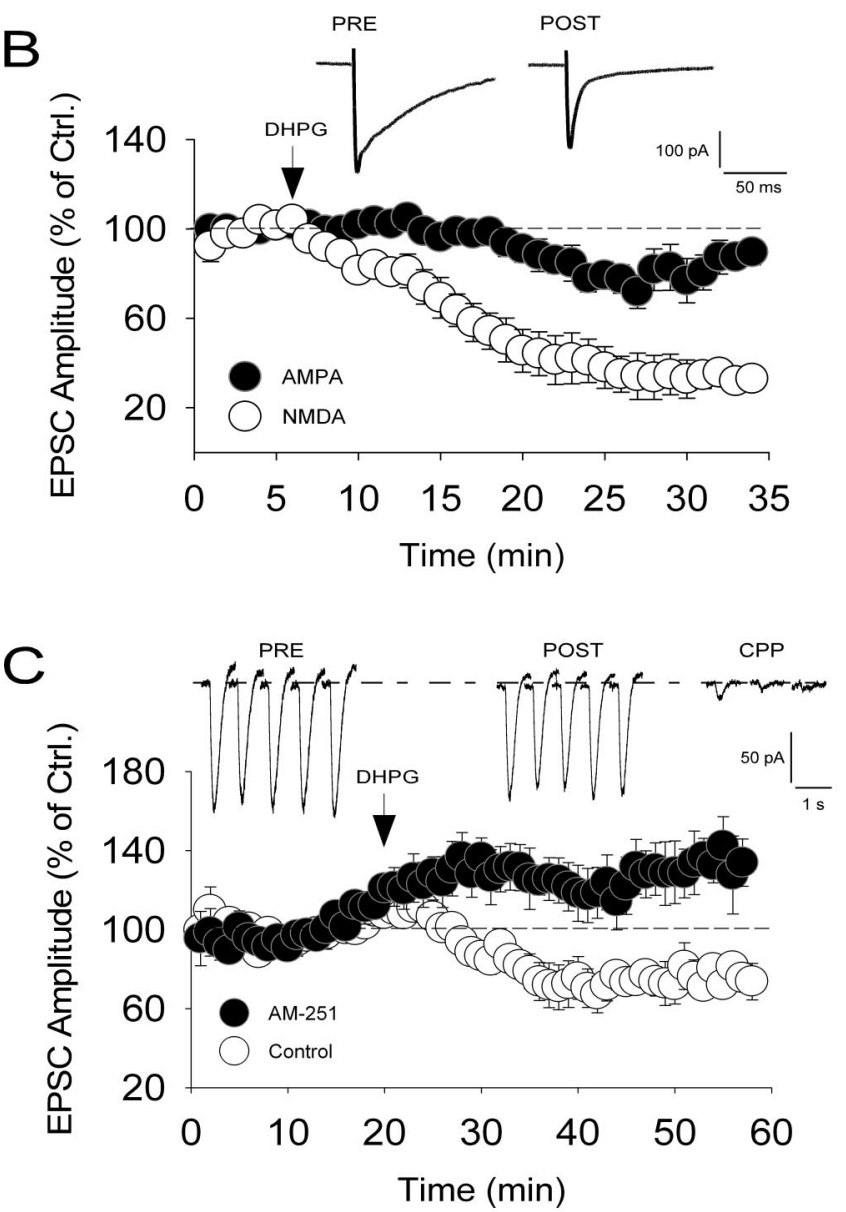

Figure 5. Postsynaptic expression of LTD. $\boldsymbol{A}$, HFS induced a stronger decrease in the NMDA (white circles) than in the AMPA (black circles) components of EPSCs. Top traces are an average of $20-50$ single traces normalized to the peak of the AMPA current. $\boldsymbol{B}$, Bath application of 50 $\mu \mathrm{M}$ DHPG after a 6 min baseline recording (indicated by arrow) induced a greater depression of the NMDA component (white circles) over the AMPA component (black circles). Average traces are shown above. DHPG was applied for the remainder of the experiment. C, DHPG application induced a reduction in NMDA currents evoked by pressure-ejected glutamate (white circles; $n=5$ ). Glutamate was puffed onto the recorded cell every $20 \mathrm{~s}$. DHPG application started after $\sim 18 \mathrm{~min}$ of baseline recording. Bath application of AM-251 prevented this depression (black circles; $n=5$ ). Upper traces are an average of 12 single traces, with each average representing 4 min of recording before (left) and after (middle) DHPG application, and after the NMDAR antagonist CPP completely eliminated the evoked responses (right). alysis of the $\mathrm{G}_{\mathrm{i} / \mathrm{o}}$-type G-protein blocker pertussis toxin (PTX). The concentration of PTX used in this study $(200 \mathrm{ng} / \mathrm{ml})$ was higher than in other reports (Hall et al. 2001). Hall et al. (2001) referred to changes in the amount of holding current and instability of membrane potential for PTX concentrations $>50 \mathrm{ng} / \mathrm{ml}$. We did not observe such effects in our recordings. Unlike group I mGluRs, CB1Rs are coupled to $\mathrm{G}_{\mathrm{i} / \mathrm{o}}$-protein. On breaking the seal with a pipette loaded with PTX, we observed a progressive increase in postsynaptic currents (Fig. $6 C)(n=5)$, with a higher increase in NMDAR than in AMPAR currents (Fig. 6C). This effect developed and reached stability over $\sim 20-25 \mathrm{~min}$. Intracellular PTX prevented the effect of WIN on the postsynaptic NMDAR currents (Fig. 6A) $(n=5)$. WIN was applied $20 \mathrm{~min}$ into the recording, to provide enough time for the PTX to reach maximum effect. This effect was mimicked by intracellular dialysis with GDP- $\beta$ S, a broad G-protein blocker (Fig. $6 B)(n=4)$, further indicating that the WIN-induced depression of NMDAR currents is mediated by G-proteins. To confirm the involvement of postsynaptic $\mathrm{G}_{\mathrm{i} / \mathrm{o}}$ in LTD, we tested the effect of intracellular PTX in HFS-induced LTD. After the evoked currents had reached a plateau, HFS was applied to induce LTD (Fig. $6 D)(n=5)$. Although we were able to induce LTD in the presence of PTX, no decrease in NMDA/AMPA ratio was observed (Fig. 6D). These results suggest that postsynaptic CB1Rs coupled to $\mathrm{G}_{\mathrm{i} / \mathrm{o}}$ are involved in the postsynaptic expression of the LTD.

Presynaptic CB1R activation decreases release probability by inhibiting the cAMP/protein kinase A (PKA) molecular pathway (Chevaleyre et al., 2007). In fact, PKA has been implicated in several forms of synaptic plasticity (Otmakhova et al., 2000; Yasuda et al., 2003; Huang and Kandel, 1994) and in the modulation of postsynaptic NMDAR currents (Skeberdis et al., 2006). If postsynaptic CB1Rs modulate NMDAR currents via PKA, intracellular dialysis with a PKA inhibitor should decrease NMDAR currents and/or occlude the changes in the NMDA/AMPA ratio induced by HFS. We found that PKA inhibitor PKI 6-22 (PKI) elicited a rapid and sharp reduction of NMDA/AMPA ratios (Fig. $7 A)(n=5)$ that occluded the effect of HFS on postsynaptic NMDAR currents (Fig. $7 B)(n=5)$. These results further support the hypothesis that postsynaptic CB1Rs mediate the postsynaptic expression of LTD.

Together, our results show that HFS in the chicken inferior colliculus triggers an LTD mediated by both presynaptic and postsynaptic mechanisms. The presynaptic effect consists of a reduction in release probability, which requires both NMDAR and CB1R activation. The postsynaptic effect is a preferential decrease of NMDAR currents mediated by CB1Rs and PKA inhibition on the postsynaptic cell.

\section{Discussion}

Although the auditory midbrain has been linked to experience dependent plasticity in birds and mammals (King et al., 1988) (for review, see Knudsen, 2002), the synaptic bases of this function are still to be determined. In fact, evidence of long-term synaptic plasticity is scarce in the auditory midbrain (Hosomi et al., 1995; Zhang and $\mathrm{Wu}, 2000$; Wu et al., 2002). Here, we show that the afferent input to EX neurons in chicken embryos undergoes an eCB-dependent LTD. LTDs mediated by CB1R activation are most frequently expressed presynaptically (Lovinger, 2008), although postsynaptic forms have also been reported (Safo and Regehr, 2005). We found simultaneous changes in the pre- and the postsynaptic compartments mediated by CB1Rs.

Bath-applied D-AP5 but not intracellular MK-801 blocks LTD, indicating that nonpostsynaptic, presumably presynaptic, 
A

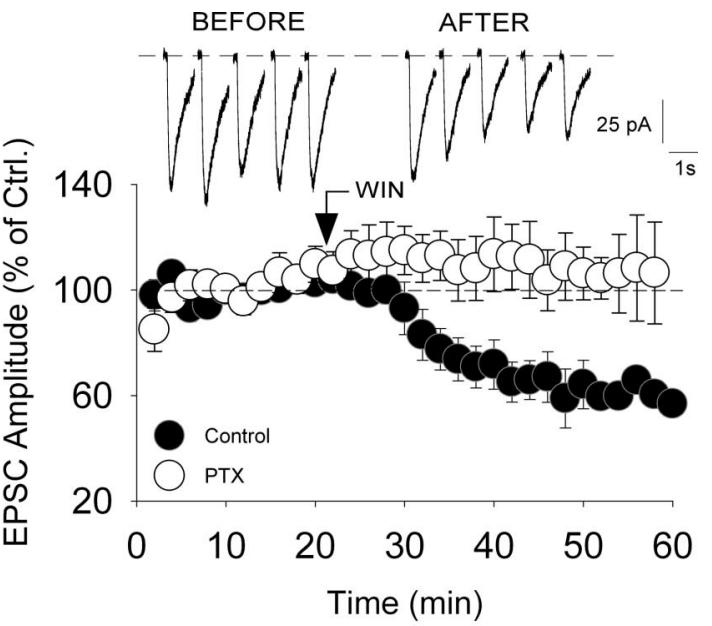

B

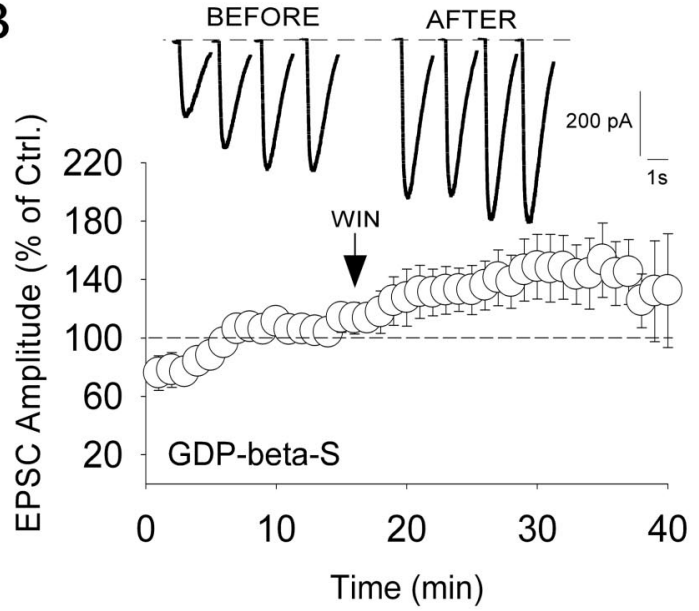

C

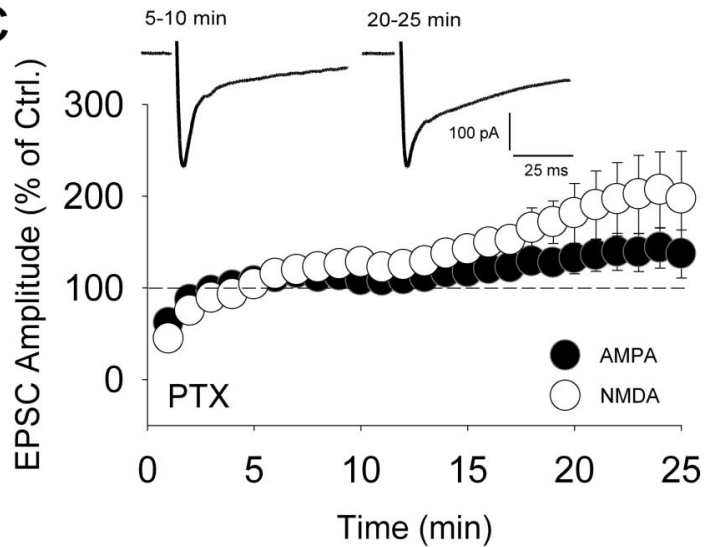

$\mathrm{D}$

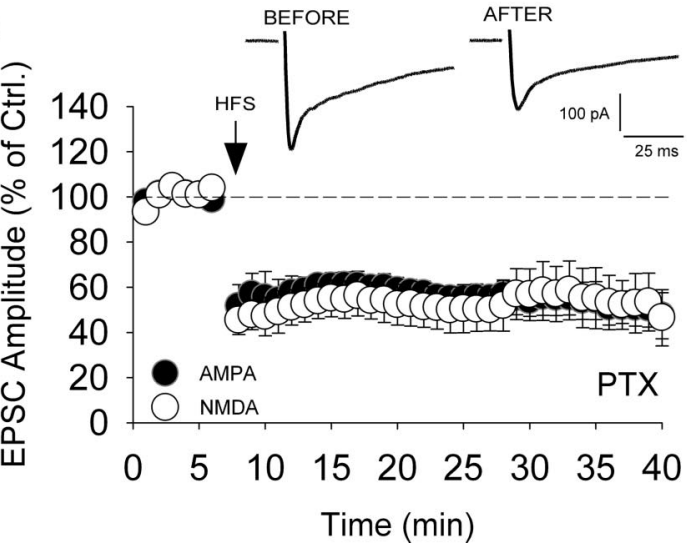

NMDARs are necessary for induction (Sjöström et al., 2003; Bender et al., 2006). Because MK-801 was applied to the recorded neuron only, NMDARs in other postsynaptic cells might be involved in triggering LTD. However, two of our findings suggest that the induction of this LTD takes place in the single cell being recorded: intracellular dialysis with BAPTA prevents LTD induction and minimal stimulation is sufficient to elicit depression. The ability of glutamate-uptake inhibitors to facilitate the generation of LTD with low-frequency stimulation is consistent with glutamate spillout activating presynaptic NMDARs. However, the exact location of these receptors remains to be determined. It is noteworthy that bath application of D-AP5 not only blocks the LTD induced by HFS but also results in a transient potentiation of synaptic currents. This phenomenon is mediated by nonpostsynaptic NMDAR activation, as it is not elicited when postsynaptic NMDARs are blocked by MK-801. Thus, presynaptic NMDARs may play a role in changing the polarity of synaptic plasticity. Similar bidirectional plasticity has been reported for calcium chelators in the cerebellum (Coesmans et al., 2004).

CB1Rs are among the most ubiquitous G-protein-coupled receptors in the brain (Herkenham et al., 1990). When activated, CB1Rs mediate a substantial decrease in neurotransmitter release (Schlicker and Kathmann, 2001) and the induction of both shortterm (Llano et al., 1991; Kreitzer and Regehr, 2001; OhnoShosaku et al., 2001; Wilson and Nicoll, 2001) and long-term forms of synaptic depression (Gerdeman et al., 2002; Marsicano et al., 2002; Chevaleyre and Castillo, 2003; Sjöström et al., 2003). Our data indicate that CB1Rs are expressed on both sides of the chicken CSh-EX synapse and together modulate synaptic function.

\section{Modulation of the presynaptic compartment by eCB}

Presynaptic NMDARs have been linked to LTD in several synapses (Lien et al., 2006; Corlew et al., 2007; Sjöström et al., 2007; Rodríguez-Moreno and Paulsen, 2008). In some cases, this process is independent of postsynaptic activity (Humeau et al., 2003). In others, postsynaptic activation is necessary (Duguid and Sjöström, 2006). Here we found an example of the latter, because postsynaptic dialysis with BAPTA completely blocks LTD. The requirement of coincident activity at the postsynaptic and presynaptic compartments may confer specificity for synaptic modulations underlying learning processes. During early life, the EX of barn owls undergoes an activity dependent refinement of its afferent inputs, which requires an instructive signal (Knud-

Figure 6. Involvement of postsynaptic CB1Rs in the reduction of NMDAR currents. $A$, WIN application reduced NMDAR currents evoked by pressure-ejected glutamate (black circles; $n=$ 5). WIN was applied to the bath solution $\sim 20$ min into the recording. Intracellular dialysis with PTX prevented this depression (white circles; $n=5$ ). Upper traces are an average of 12 single traces, with each average representing 4 min of recording before (left) and after (right) WIN application. $\boldsymbol{B}$, Same procedure as in $\boldsymbol{A}$ with GDP- $\beta$ S application instead of PTX. WIN was applied $\sim 16$ min into the recording. Upper traces are an average of 12 single traces, with each average representing $4 \mathrm{~min}$ of recording before (left) and after (right) WIN application. $\boldsymbol{C}$ Intracellular dialysis with PTX ( $200 \mathrm{ng} / \mathrm{ml})$ induced a run-up of both AMPA (black circles) and NMDA (white circles) currents. Current amplitudes were normalized to the mean amplitude over the first 10 min of recording. Upper traces are averages of 60 single traces computed between 5 and 10 min (left) and $20-25$ min (right) into the recording, normalized to the peak of the AMPA current. $D$, Same neurons shown in $\boldsymbol{B}$, after both AMPA and NMDA currents reached a plateau. The NMDA component was normalized to the AMPA component for the last 6 min and HFS stimulation was applied to induce LTD. HFS with intracellular dialysis of PTX induced LTD by depressing both the NMDA (white circles) and AMPA (black circles) components without affecting the NMDA/AMPA ratio. Average traces of all the single traces recorded before and after HFS are shown above. 
sen 2002). To produce an accurate map of auditory space, precise regulation of synaptic inputs is needed (DeBello et al., 2001).

\section{Modulation of the postsynaptic compartment by eCB: reduction of NMDAR currents}

The long-term reduction in NMDAR currents after LTD induction indicates that a change in NMDAR function takes place concomitantly with the decrease in release probability. This idea is supported by our finding that NMDAR currents induced by focal injection of glutamate decrease after DHPG application. This effect is mediated by CB1Rs because it can be blocked by AM-251 and be mimicked by bath application of WIN. The involvement of $\mathrm{G}_{\mathrm{i} / \mathrm{o}}$ and PKA in the postsynaptic expression of LTD suggests that CB1Rs mediating changes in NMDA/AMPA ratios are located in the postsynaptic compartment.

To date, no report has shown long-term decrease in postsynaptic NMDAR currents mediated by eCBs. It remains to be shown, whether the decrease in NMDAR currents is caused by a change in NMDAR availability or function. Because changes in NMDAR currents are not measured at the hyperpolarizing potentials generally used in studies of synaptic plasticity, it is possible that this form of coincident plasticity is present in other systems but has not been detected. Kwon and Castillo (2008) showed an enhancement of postsynaptic NMDA transmission after presynaptic long-term potentiation in the mossy fiber to CA3 pyramidal cell synapse of the hippocampus. Our results indicate that the long-term changes in postsynaptic NMDA conductance may be mediated by postsynaptic CB1Rs. As such, and pending confirmation with electron microscopy, this represents novel evidence suggesting that eCBs act on the presynaptic and postsynaptic compartments independently.

In barn owls, the responses of EX neurons that are suppressed by experience contain a lower NMDA/AMPA ratio than newly acquired ones (Feldman and Knudsen, 1998). Furthermore, a recent report showed that cAMP response element binding protein (CREB), a downstream target of the cAMP/PKA pathway, is involved in remapping the inferior colliculus during prisminduced learning in barn owls (Nichols and DeBello, 2008). Our result that LTD at the CSh-EX synapse is mediated by a PKAdependent reduction of the NMDA/AMPA ratio is consistent with these findings. The synaptic mechanism described here could thus be relevant for the input remapping observed in vivo.

\section{References}

Bender VA, Bender KJ, Brasier DJ, Feldman DE (2006) Two coincidence detectors for spike timing-dependent plasticity in somatosensory cortex. J Neurosci 26:4166-4177.

Brainard MS, Knudsen EI (1993) Experience-dependent plasticity in the inferior colliculus: a site for visual calibration of the neural representation of auditory space in the barn owl. J Neurosci 13:4589-4608.

Chevaleyre V, Castillo PE (2003) Heterosynaptic LTD of hippocampal GABAergic synapses: a novel role of endocannabinoids in regulating excitability. Neuron 38:461-472.

Chevaleyre V, Takahashi KA, Castillo PE (2006) Endocannabinoidmediated synaptic plasticity in the CNS. Annu Rev Neurosci 29:37-76.

Chevaleyre V, Heifets BD, Kaesser PS, Südhof TC, Purpura DP, Castillo PE

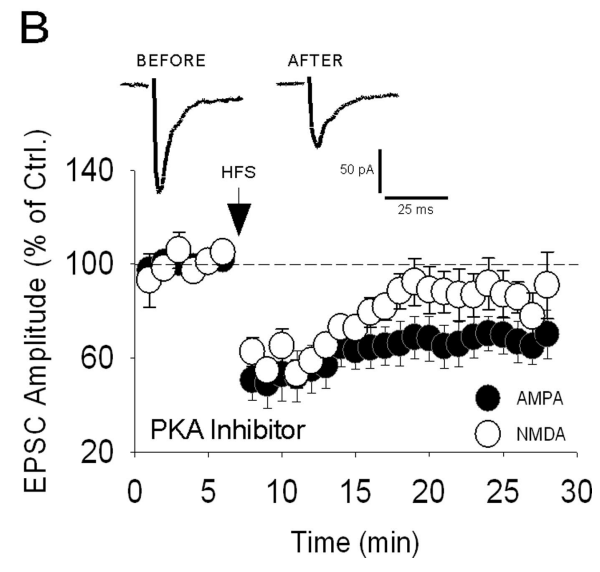

Figure 7. PKA inhibition occludes postsynaptic LTD. $\boldsymbol{A}$, Intracellular dialysis with the PKA blocker PKI $6-22$ (PKI; $10 \mu \mathrm{M})$ reduced NMDA/AMPA ratios to ranges similar to control measurements using HFS. HFS with PKI in the pipette solution did not A and after HFS.

(2007) Endocannabinoid-mediated long-term plasticity requires cAMP/ PKA signaling and RIM1alpha. Neuron 54:801-812.

Coesmans M, Weber JT, De Zeeuw CI, Hansel C (2004) Bidirectional parallel fiber plasticity in the cerebellum under climbing fiber control. Neuron 44:691-700.

Coles RB, Aitkin LM (1979) The response properties of auditory neurons in the midbrain of the domestic fowl (Gallus gallus) to monaural and binaural stimuli. J Comp Physiol 134:241-251.

Corlew R, Wang Y, Ghermazien H, Erisir A, Philpot BD (2007) Developmental switch in the contribution of presynaptic and postsynaptic NMDA receptors to long-term depression. J Neurosci 27:9835-9845.

DeBello WM, Feldman DE, Knudsen EI (2001) Adaptive axonal remodeling in the midbrain auditory space map. J Neurosci 21:3161-3174.

Duguid I, Sjöström PJ (2006) Novel presynaptic mechanisms for coincidence detection in synaptic plasticity. Curr Opin Neurobiol 16:312-322.

Feldman DE, Knudsen EI (1998) Pharmacological specialization of learned auditory responses in the inferior colliculus of the barn owl. J Neurosci 18:3073-3087.

Gerdeman GL, Ronesi J, Lovinger DM (2002) Postsynaptic endocannabinoid release is critical to long-term depression in the striatum. Nat Neurosci 5:446-451.

Hall KE, Liu J, Sima AA, Wiley JW (2001) Impaired inhibitory G-protein function contributes to increased calcium currents in rats with diabetic neuropathy. J Neurophysiol 86:760-770.

Herkenham M, Lynn AB, Little MD, Johnson MR, Melvin LS, de Costa BR, Rice KC (1990) Cannabinoid receptor localization in brain. Proc Natl Acad Sci U S A 87:1932-1936.

Hosomi H, Hirai H, Okada Y, Amatsu M (1995) Long-term potentiation of neurotransmission in the inferior colliculus of the rat. Neurosci Lett 195:175-178.

Huang YY, Kandel ER (1994) Recruitment of long-lastin and protein kinase A-dependent long term potentiation in the CA1 region of hippocampus requires repeated tetanization. Learn Mem 1:74-82.

Humeau Y, Shaban H, Bissière S, Lüthi A (2003) Presynaptic induction of heterosynaptic associative plasticity in the mammalian brain. Nature 426:841-845.

Kaslow HR, Burns DL (1992) Pertussis toxin and target eukaryotic cells: binding, entry, and activation. FASEB J 6:2684-2690.

King AJ, Hutchings ME, Moore DR, Blakemore C (1988) Developmental plasticity in the visual and auditory representations in the mammalian superior colliculus. Nature 332:73-76.

Knudsen EI (2002) Instructed learning in the auditory localization pathway of the barn owl. Nature 417:322-328.

Knudsen EI, Knudsen PF (1989) Vision calibrates sound localization in developing barn owls. J Neurosci 9:3306-3313.

Kreitzer AC, Regehr WG (2001) Retrograde inhibition of presynaptic calcium influx by endogenous cannabinoids at excitatory synapses onto Purkinje cells. Neuron 29:717-727. 
Kwon HB, Castillo PE (2008) Long-term potentiation selectively expressed by NMDA receptors at hippocampal mossy fiber synapses. Neuron 57:108-120.

Lien CC, Mu Y, Vargas-Caballero M, Poo MM (2006) Visual stimuliinduced LTD of GABAergic synapses mediated by presynaptic NMDA receptors. Nat Neurosci 9:372-380.

Llano I, Leresche N, Marty A (1991) Calcium entry increases the sensitivity of cerebellar Purkinje cells to applied GABA and decreases inhibitory synaptic currents. Neuron 6:565-574.

Lovinger DM (2008) Presynaptic modulation by endocannabinoids. Handb Exp Pharmacol 435-477.

Marsicano G, Wotjak CT, Azad SC, Bisogno T, Rammes G, Cascio MG, Hermann H, Tang J, Hofmann C, Zieglgänsberger W, Di Marzo V, Lutz B (2002) The endogenous cannabinoid system controls extinction of aversive memories. Nature 418:530-534.

Massey PV, Johnson BE, Moult PR, Auberson YP, Brown MW, Molnar E, Collingridge GL, Bashir ZI (2004) Differential roles of NR2A and NR2B-containing NMDA receptors in cortical long-term potentiation and long-term depression. J Neurosci 24:7821-7828.

Nichols GS, DeBello WM (2008) Bidirectional regulation of the cAMP response element binding protein encondes spatial map alignment in prism adapting barn owls. J Neurosci 28:9898-9909.

Ohno-Shosaku T, Maejima T, Kano M (2001) Endogenous cannabinoids mediate retrograde signals from depolarized postsynaptic neurons to presynaptic terminals. Neuron 29:729-738.

Otmakhova NA, Otmakhov N, Mortenson LH, Lisman JE (2000) Inhibition of the cAMP pathway decreases early long-term potentiation at CA1 hippocampal synapses. J Neurosci 20:4446-4451.

Patneau DK, Mayer ML (1990) Structure-activity relationship for amino acid transmitter candidates acting at $N$-methyl-D-aspartate and quisqualate receptors. J Neurosci 10:2385-2399.

Puelles L, Martinez-de-la-Torre M, Paxinos G, Watson C, and Martinez S (2007) The chick brain in stereotaxic coordinates. New York: Academic.

Rodriguez-Contreras A, Liu XB, DeBello WM (2005) Axodendritic contacts onto calcium/calmodulin-dependent protein kinase type II-expressing neurons in the barn owl auditory space map. J Neurosci 25:5611-5622.

Rodríguez-Moreno A, Paulsen O (2008) Spike timing-dependent longterm depression requires presynaptic NMDA receptors. Nat Neurosci 11:744-745.

Safo PK, Regehr WG (2005) Endocannabinoids control the induction of cerebellar LTD. Neuron 48:647-659.

Schlicker E, Kathmann M (2001) Modulation of transmitter release via presynaptic cannabinoid receptors. Trends Pharmacol Sci 22:565-572.

Sjöström PJ, Turrigiano GG, Nelson SB (2003) Neocortical LTD via coincident activation of presynaptic NMDA and cannabinoid receptors. Neuron 39:641-654.

Sjöström PJ, Turrigiano GG, Nelson SB (2007) Multiple forms of long-term plasticity at unitary neocortical layer 5 synapses. Neuropharmacology 52:176-184.

Skeberdis VA, Chevaleyre V, Lau CG, Goldberg JH, Pettit DL, Suadicani SO, Lin Y, Bennett MV, Yuste R, Castillo PE, Zukin RS (2006) Protein kinase A regulates calcium permeability of NMDA receptors. Nat Neurosci 9:501-510.

Wilson RI, Nicoll RA (2001) Endogenous cannabinoids mediate retrograde signalling at hippocampal synapses. Nature 410:588-592.

Wilson RI, Nicoll RA (2002) Endocannabinoid signaling in the brain. Science 296:678-682.

Wu SH, Ma CL, Sivaramakrishnan S, Oliver DL (2002) Synaptic modification in neurons of the central nucleus of the inferior colliculus. Hear Res 168:43-54.

Yang CH, Huang CC, Hsu KS (2005) Behavioral stress enhances hippocampal CA1 long-term depression through the blockade of the glutamate uptake. J Neurosci 25:4288-4293.

Yasuda H, Barth AL, Stellwagen D, Malenka RC (2003) A developmental switch in the signaling cascades for LTP induction. Nat Neurosci 6:15-16.

Zhang Y, Wu SH (2000) Long-term potentiation in the inferior colliculus studied in rat brain slice. Hear Res 147:92-103.

Zucker RS, Regehr WG (2002) Short-term synaptic plasticity. Annu Rev Physiol 64:355-405. 